\title{
Molecular Modeling of the Chiral Recognition of Propranolol Enantiomers by a $\beta$-Cyclodextrin
}

\author{
Hyunmyung Kim, Karpjoo Jeong, ${ }^{\dagger}$ Sangsan Lee, ${ }^{\ddagger}$ and Seunho Jung ${ }^{\star}$ \\ Department of Aicrobial Engineering and Bio Holecular Informatics Center, ${ }^{\dagger}$ College of Information and Communication, \\ Konktk Linversity, I Hwanang-dong, Gwangin-gu, Seoul 143-701, Korea

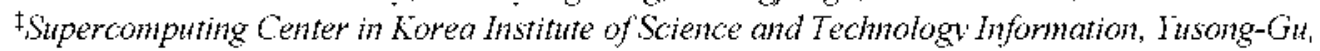 \\ Eoeth-Dong 52, Daejon 305-806, Korea \\ Received October 20,2002
}

\begin{abstract}
Enantioselectivity of the propranolol on $\beta$-cyclodextrin was simulated by molecular modeling. Monte Carlo (MC) docking and molecular dy namics (MD) simulations were applied to investigate the molecular mechanism of enantioselective difference of both enantiomeric complexes. An energetic analysis of MC docking simulations coupled to the $\mathrm{MD}$ simulations successfully explains the experimental elution order of propranolol enantiomers. Molecular dynamics simulations indicate that average energy difference between the enantiomeric complexes. frequently used as a measure of chiral recognition. depends on the length of the simulation time. We found that. only in case of much longer $\mathrm{MD}$ simulations. noticeable chiral separation was observed
\end{abstract}

Key Words : Chiral discrimination, Monte Carlo docking, Molecular dynamics sinulations. $\beta$-Cyclodestrin. Propranolol

\section{Introduction}

Chiral discrimination has been a subject of great interest in the development. use. and action of phamaceutical agent. Most often the enantiomers of chiral drugs have different pharmacological and toxicological properties and therefore the quantitative enantiomeric composition of these drugs should be determined. ${ }^{3}$ Propranolol (1-isopropy lamino-3-(1naphthyloxy)-2-propranolol: Figure 1) is an important $\beta$ adrenergic blocking agent which has gained widespread usage in the treatment of angina pectoris, cardiac dy srhythmias and hypertension. The pharmacological properties of the enantiomers of propranolol are quite different. and the $\beta$ adrenergic blocking activity resides in the $(S)-(-)$ isomer while the $(R)-(+)$-enantiomer has only a membrane stabilizing effect -5 Further. the hepatic oxidation of propranolol is highly stereospecific. ${ }^{3}$ It is therefore important to have a method for the precise and accurate determination of the enantiomeric purity.

An inportant method for separating enantioners involves cyclodextrins ${ }^{6} \beta$-Cyclodextrin $(\beta-C D)$ is a macrocyclic molecule formed by $\alpha-(\mathrm{l} \rightarrow 4)$ glycosidic links between

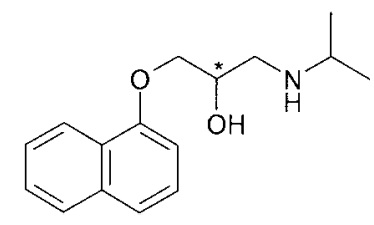

Figure 1. Chemical structure of propranolol. The chiral carbon is indicated by asterisk.

Corresponding Author: Phone \& Fax: $+82-2-450-3520$, e-mail: shjung atkonkuk.ac.kT seven D-glucose monomer units. adopt a toroid shape. The resulting cavity of the cyclodextrins well characterized complexing properties with the appropriate guest molecules. ${ }^{7}$ The inherent chirality of the cyclodextrin molecules allows them to form diastereomeric complexes with enantiomeric compounds. Thus, it has been used as bonded chiral phases in liquid cluromatography (LC) or as chiral mobile phase additives in LC and capillary electophoresis (CE) for the enantiomeric separation of racemic molecules. ${ }^{1.8}$

In this study. the inclusion complexes fonmed between $\beta$ $C D$ and both propranolol enantiomers were modeled and refined by molecular modeling methods to correctly predict the elution order for enantiomeric separations. The interaction energies and conformation of both propranolol- $\beta-C D$ complexes were compared.

\section{Experimental Sections}

Modeling host and guests molecules. Molecular mechanics and dynamics calculations were performed with the InsightII/ Discover program (version 2000, Molecular Simulations Inc. San Diego. U.S.A.) using consistent valence force field (CVFF) on a SGI OCTANE 2 workstation (Silicon Graphics. U.S.A.). The $\beta$-CD structure was obtained by energy minimization of a crystallographic geometry. ${ }^{16}$ The conformational search of (R)- and (S)-propranolol were performed by simulated annealing molecular dynamics-full energy minimization strategy.11.12 and the lowest energy conformation of each enantiomer was selected for further simulations. The conformations of these molecules are depicted in Figure 2.

Monte Carlo docking minimization simulations. The host and guest molecules were positioned in the neighborhood with a distance of $\sim 15 \AA^{13}$ Monte Carlo docking 
(A)
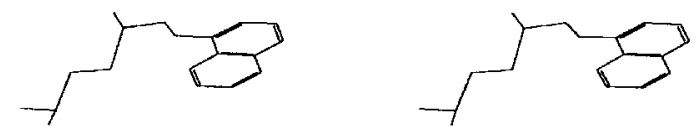

(B)
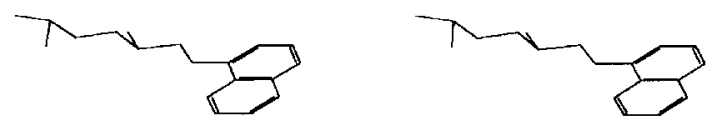

(C)
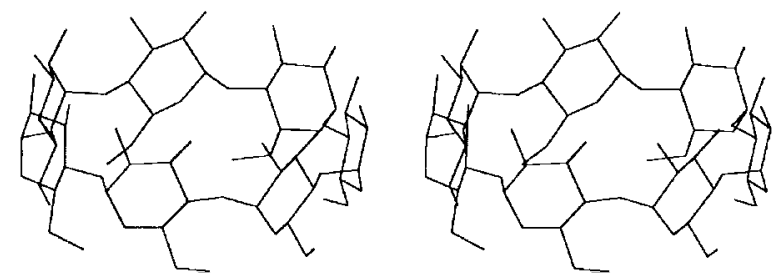

Figure 2. Stereoview of molecular models used in the $\mathrm{MC}$ simulations. (A) (R)-propranolol, (B) (S)-propranolol, (C) $\beta$-CD.

simulations started by conjugate gradient energy minimization of this initial configuration for 100 iterations and accepted it as the first frame. In the course of trial to a new configuration. propranolol could take translational movement of maximum $3 A$ to $x, y$ and $z$ axis and rotation of maximum $180^{\circ}$ around $\mathrm{x}, \mathrm{y}$ and $\mathrm{z}$ axis. Total 6 degrees of freedom was present for this sy'stem (3 translational. 3 rotational). Each cycle began with a random change of up to 5 degrees of freedom among them. ${ }^{14}$ If the energy of the resulting configuration was within $10000 \mathrm{kcal} / \mathrm{mol}$ from the last accepted one. it was subjected to the 100 iterations of conjugated gradient energy minimization. The energy tolerance of $10000 \mathrm{kcal} / \mathrm{mol}$ was imposed to avoid significant overlap of van der Waals radii in the random search. After the energy minimization, the resulting structure was accepted based on criteria. (a) An energy check with the Metropolis criteria at $300 \mathrm{~K}^{15}$ and (b) a root-mean-squared displacement (RMSD) check. which compared the RMSD of the new configuration against those accepted so far. Configurations within 0.1 A RMSD of pre-existing ones were discarded to avoid accepting similar configurations. The Monte Carlo simulations were performed until energy convergence. No cutoff was imposed on the calculation of non-bonded interactions. and the dielectric constant was set to 1 . Boltzmann averages of energies were evaluated at 300 $\mathrm{K}$.

Molecular dynamics simulations. We used lowest-energy structures from the MC docking simulations as starting conformations for further molecular dy'namics simulations. ${ }^{16}$ No cut-off was imposed on the calculation of non-bonded interactions. Constant NVT molecular dynamics calculations were performed using the leap-frog algorithm with a 1 fs time step. The initial atomic velocities were assigned from a Gaussian distribution corresponding to a temperature of 300 $\mathrm{K}$. The system was equilibrated for $500 \mathrm{ps}$ and the production run was done for $100 \mathrm{~ns}$. The temperature was controlled by velocity scaling in equilibration phase and by Berendsen algorithm ${ }^{17}$ in production phases with a coupling constant of $0.2 \mathrm{ps}$. Intermediate structures were saved every'
$10 \mathrm{ps}$ for analysis. The dielectric constant was set to $l$ or $\mathrm{r}$. The effects of the implicit solvent are approximated using a dielectric constant proportional to the distance $(\varepsilon=r){ }^{18}$

\section{Results and Discussion}

Monte Carlo docking minimization simulations. The pathways of MC docking simulations showed a general tendency of inclusion complex formation and lowering complexation energy: The complexation energy was defined as the difference between the sum of the energy of individual host and guest molecule and the energy of the inclusion complex. ${ }^{19}$ Figure 3 compares the complexation energies in $\mathrm{MC}$ runs for both complexes. For each enantiomer. the MC process could be divided into two phases: the initial and equilibrium phase. In the initial phase (from trial 1 to 5000 ). the complexation energies decreased rapidly. and the guest (both enantiomers) kept in contact with the host and the guest searched for stable conformations in the cavity of the host. In the equilibrium phase (from trial 5000 to the end). the complexation energies reached its equilibrium value and fluctuated around it in a stable manner.
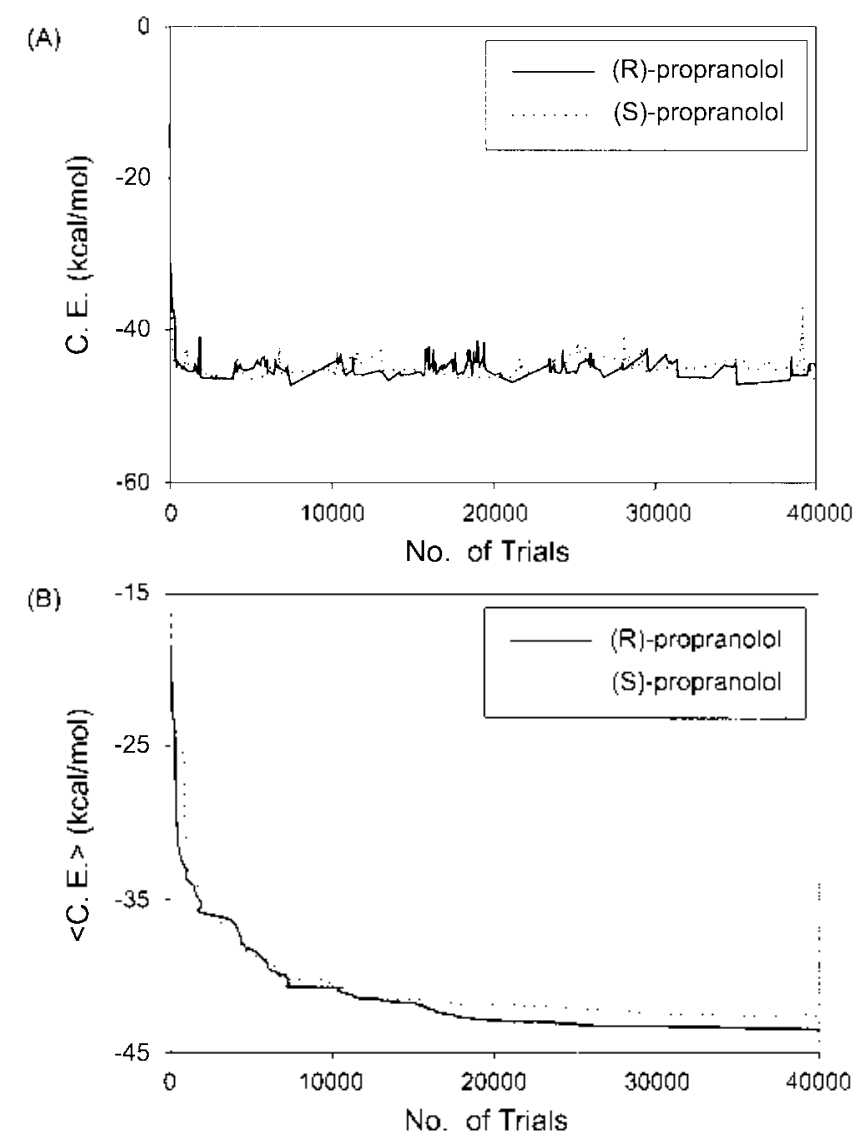

Figure 3. Energy profile of the Metropolis Monte Carlo docking simulations. The complexation energy was defined as the difference between the sum of independently calculated energy of each hostguest molecule and the energy of each configuration in the process. The average energy was evaluated using the relation, $\angle \mathrm{E}\rangle=(\mathrm{l} / \mathrm{N})$ $\Sigma \mathrm{E}_{\imath}$, where $\mathrm{N}$ is the number of trials and $\mathrm{E}_{s}$ is its energy. (A) Complexation energy, (B) Average complexation energy ( $<$ C. E $>$ ). 
(A)
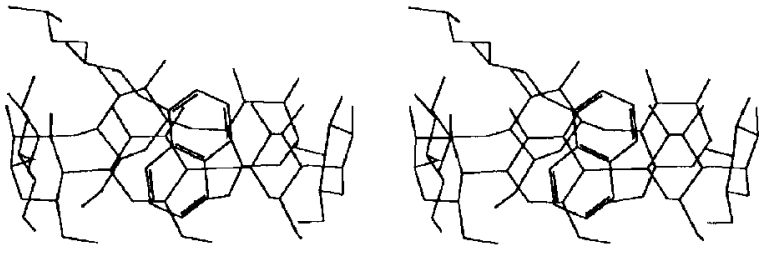

(B)

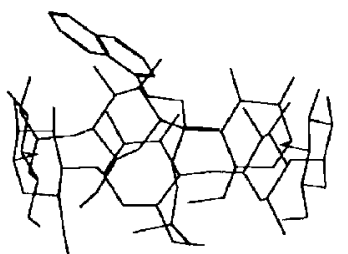

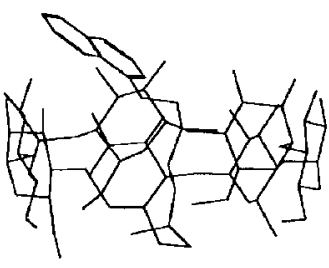

Figure 4. Stereoview of lowest-energy configurations of the inclusion complexes of both enantiomers of propranolol and $\beta-C D$ in $\mathrm{MC}$ docking simulations. (A) (R)-propranolol- $\beta$-CD complex; (B) (S)-propranolol- $\beta$-CD complex.

The average complexation energies of (R)-propranolol- $\beta$ $\mathrm{CD}$ complex are lower than that of (S)-propranolol- $\beta$-CD complex. The axerage complexation energy of the (S)propranolol- $\beta$-CD complex was $43.89 \pm 1.62 \mathrm{kcal} / \mathrm{mol}$. whereas that of the (R)-propranolol- $\beta$-CD complex was $44.43 \pm$ $1.06 \mathrm{kcal} / \mathrm{mol}$ in the equilibrium phase. A Boltzmann average of resulting complexation energies for the each $\mathrm{MC}$ docking
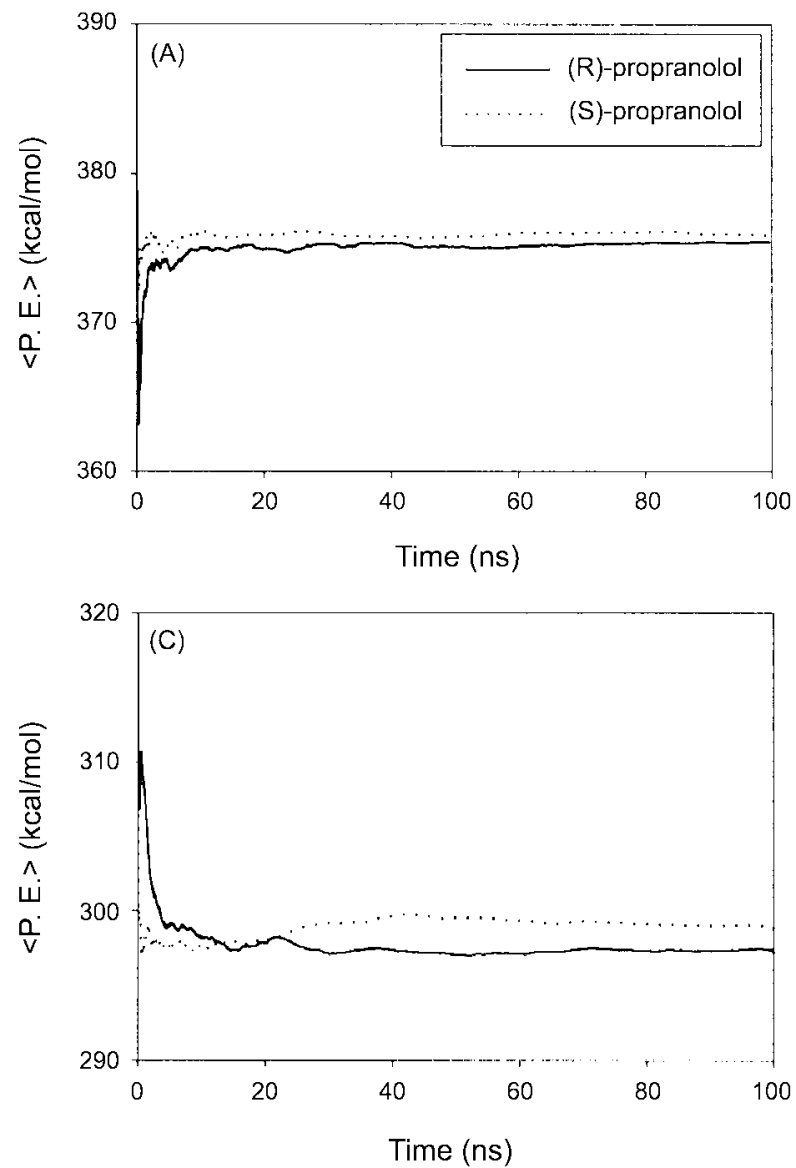

was calculated ${ }^{2(1)}$ The Boltzmann average of the (S)propranolol- $\beta$-CD complex is $45.63 \mathrm{kcal} / \mathrm{mol}$, whereas that of the (R)-propranolol- $\beta-C D$ is $-45.93 \mathrm{kcal} / \mathrm{mol}$ in equilibrium phase. The lower complexation energy of the (R)propranolol- $\beta$-CD complex could indicate the formation of thermodynamically more stable inclusion complex. The energy analysis below 20,000 trials also shows similar result (data not shown). These results are consistent with reported experimental results that $\mathrm{R}$ isomer was retained longer in the separation process than the $\mathrm{S}$ isomer. ${ }^{1.21}$ We computed the average distance between the propranolol's naphthyl moiety and the geometric center of the $\beta$-CD to assess the relative compactness of the two complexes. In order to do this effectively: a dunmy atom was defined to represent the geometric center of the naphthyl moiety. The average distance between the naphthyl moiety of propranolol and the $\beta$ $C D$ center is $2.33 \AA$ for the less tightly bound (S)propranolol and $1.09 \mathrm{~A}$ for the more tightly bound (R)propranolol. Figure 4 shows the lowest energy configuration of each inclusion complex of (R)- and (S)- propranolol with $\beta$-CD. The orientation of guest was defined as being 'up' or 'down' meaning that the naphthyl moiety was oriented toward the secondary rim or toward primary rim of $\beta$-CD. respectively. The energetic analy sis of each complex indicated the major guest orientation of R-complex was "down"
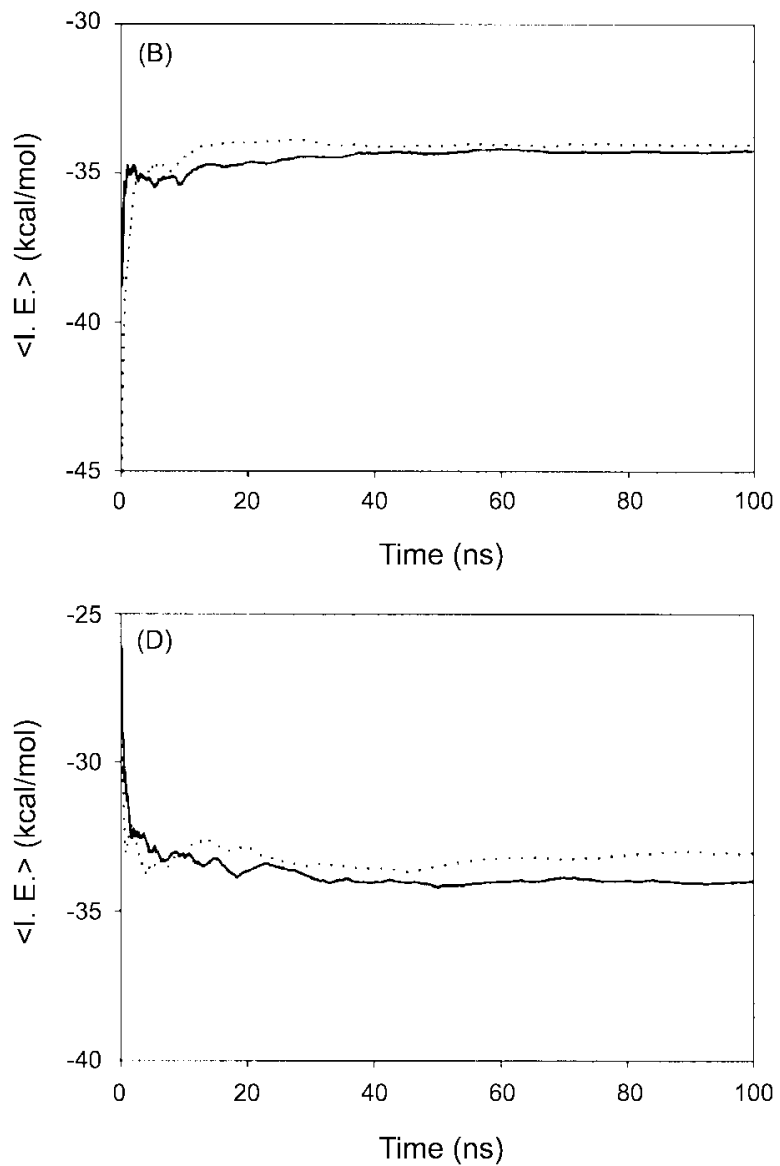

Figure 5. The average potential energy $(<\mathrm{P}$. E $>)$ and interaction energy $(<\mathrm{I} . \mathrm{E}>)$ in the $\mathrm{MD}$ simulations. The interation enlergy was defined as nonbond energies between host and guest molecule. The average energy was evaluated using the relation, $\angle \mathrm{E}>=(1 / \mathrm{N}) \Sigma \mathrm{E}_{i}$, where $\mathrm{N}$ is the number of frames and $E_{i}$ is its energy. $(A)<$. E $>$ in $\varepsilon=1,(B)<\mathrm{I}$. E $>$ in $\varepsilon=1,(D)<P$. E $>$ in $\varepsilon=r$, and (D) $<\mathrm{I}$. $E>$ in $\varepsilon=r$. 
and that of S-complex was "up". Each stable complex showed the tight inclusion complex between the hydrophobic moiety of guest and hydrophobic cavity of $\beta$-CD. ${ }^{1}$

The $\mathrm{MC}$ docking simulations are sufficiently accurate to model enantiomeric separations. thus it can serve as an effective method for selecting starting points for further investigation. Consequently: we carried out molecular dy'namics simulations to investigate the interaction energy between each host and guest over a time period. The lowest energy: conformation in $\mathrm{MC}$ docking simulations was used as an initial conformer for molecular dynamics simulations.

Molecular dynamics simulations. In most molecular dynamics studies of $\mathrm{CD}$ complexes. calculations were carried out for quite short simulation time $(<5 \mathrm{~ns}) .^{-2-23} 12 \mathrm{~ns}$ MD simulations in water was considered by Dodziuk et al. however. these systems have not reached complete equilibrium states particularly for the chiral recognition process. ${ }^{2+}$ Therefore. we have extended the MD simulations with the implicit solvent for considerably longer times. about $100 \mathrm{~ns}$. and analyzed how the calculated average energy differences between the enantiomeric complexes depend on the length of the simulation. The time change of the potential energy: and interaction energy during the MD simulations of both complexes is shown in Figure 5 . The interaction energy' was defined as nonbond energies between host and guest molecule. The average energy' was evaluated using the relation. $\langle\mathrm{E}\rangle=(1 / N) \Sigma \mathrm{E}_{i}$. where $\mathrm{N}$ is the number of frames and $\mathrm{E}_{i}$ is its energy. Figure 5 shows that $\mathrm{R}$ isomer is not always predicted to form more stable complex. Before 20 ns. the average potential energy and interaction energy was inverted. (R)-propranolol- $\beta$-CD complex kept up more stable for the rest of simulation. Energy difference in $\varepsilon=r$ (the effects of the implicit solvent) is higher than in $\varepsilon=1$ ( $\Delta$ I.E.S.R $=0.95$ $\mathrm{kcal} / \mathrm{mol}$ in $\varepsilon=\mathrm{r}$. $\Delta$ I.E.s. $\mathrm{R}=0.22 \mathrm{kcal} / \mathrm{mol}$ in $\varepsilon=1$ ). These results strongly indicate that solvation effect is one of the important parts of chiral recognition process by $\beta-\mathrm{CD}$. These MD simulations correctly predict the $\mathrm{R}$ isomer to be bound tighter. These results also indicate that much longer MD simulations times must be applied in order to get the reliable results of for enantiomeric separation.

\section{Conclusion}

Throughout this study. we investigated the molecular models of chiral discrimination by $\beta-C D$ through the differences in the interaction energies and configuration of inclusion complexes by molecular modeling. The calculated results are in agreement with experimental observation in predicting the correct elution order in propranolol separation. Molecular dynamics simulations indicate that average energy difference between the complexes. which is frequently used as a measure of chiral recognition $\cdots$ the length of the simulation time. We found that only in case of much longer MD simulations. more than 10 times for the general MD time scale. noticeable chiral separation was observed. Our results suggest that molecular modeling methods such as MC and 100 ns MD simulations successfully' explained the experimental results about the chiral recognition process of propranolol by $\beta$-CD. We hope that our suggested methods would be applicable for a variety of other chiral separation șystemıs.

Acknowledgment. This study was supported by a grant of the Korea Health 21 R\&D Project. Ministry of Health \& Welfare. Republic of Korea. (02-PJ1-PG3-51103-0001). SDG.

\section{References}

1. Amstrong. D. W: Ward, T. J: Armstrong. R. D.: Beesley: T. E. Science 1986. 232. 1132

2. Potter. L. T. J. Phamacol. Exp. Ther: 1967. 155.91.

3. Walle. T.: Walle. U. K.: Wilson. M. T.: Fagan. T. C.: Gafney. T. E. Br. J. Chin. Phormacol 1984, 18,741.

4. Rahn. K. H.: Hawlina, A.; Kersting, F: Planz. G. NammSchmedeberg's Arch. Pharnacol 1974, 286,319.

5. Howe. R.: Shanks. R. G. Nattre 1966. 210. 1336.

6. Redondo. . .: Blazquez. M. A.: Torrens. A. Chiralin 1999. 11.694

7. Hamilton1. T. A.: Chen1. L. J. Am. Chem. Soc. 1988. 110. 5833.

8. Berthod. A.: Jin. H. L.; Beesley, T. E.; Duncan, J. D.: Armstrong, D. W. J. Pharnt Bionted Anal 1990. 8. 123.

9. Dauber-Osguthorpe, P; Roberts, V. A.; Osguthorpe. D. J.: Wolff, J.: Genest. M.: Hagler. A. T. Proteins 1988. 4. 31.

10. Betzel. C.: Saenger. W.: Hingerty. B. E.: Brown. G. M. J. Am Chem. Soc. 1984. 106.7545.

11. Choi. Y: Kang. S.: Yang, C.-H.: Kim. H.-W.: Jung. S. Bull. Korean Chem. Soc. 1999. 20.753.

12. Choi. Y.: Yang, C.-H; Kim, H.W: Jung. S. Corbolndt Res 2000. 326.227 .

13. Chio. Y: Yang. C.-H.: Kiml. H.W.: Jung. S. Carbolydr: Res. 2000. 328.393 .

14. Hart. T. N.; Read. R. J. Proteins 1992. 13, 206

15. Metropolis. N.: Rosenbluth. A. W.: Rosenbluth. M. N.: Teller, A. H. Teller. E. J. Chent. Phns. 1953, 21. 1087.

16. Chio. Y: Yang, C.-H.: Kim. H.W. Jung, S. J. Inct Phenon. 2001.39.71.

17. Berendsen. H. I. C.: Postma. T. P. M.: van1 Gunsteren. W. F.: DiNola. A.: Haak. J. R. J. Chem. Plys. 1984. 81. 3684.

18. Luty. B. A.; Wasserman, Z. R.: Stouten, P. F. W.: Hodge. C. N.: Zacharias, M.; MoCammon. J. A. J. Conp. Chem. 1995, 16. 454.

19. Kim, H.: Choi, J; Kim. H.W: Jung. S. Carbohldr. Res. 2002. 337. 549 .

20. Black. D. R.: Parker. C. G.: Zimmeman. S. S.: Lee. M. L. J. Comp. Chem. 1996.17.931.

21. Pham-Huy: C.: Radenen, B.; Sahui-Gnassi. A.: Claude. J. J. Chrontatogr: $B$ 1995, 665. 125.

22. Dodziuk. H: Ejchart. A: Lukin, O: Vysotsky. M. O. J. Ong. Chem 1999.61. 1503

23. Lipkowitz. K. B.: Stcehr. C. M. Chirality 1996. 8. 341

24. Dodziuk. H.: Lukin. O. Chem. Phys. Lett. 2000. 327. 18

25. Ramirez. J.: Ahn, S.; Grigorean. G; Lebrilla. C. B. J. Am. Chent Soc. $2000,122,6884$. 